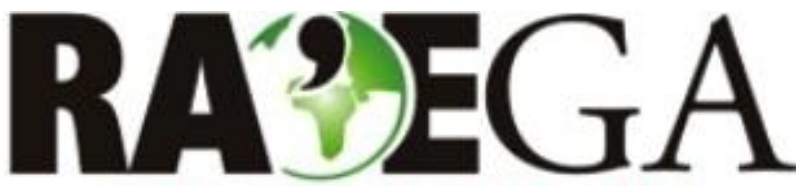

O ESPACO GEOGRÁFICO EM ANÁLISE

\title{
AVALIAÇÃO DO RUÍDO DE TRÁFEGO NOTURNO - ESTUDO DE CASO NA CIDADE DE CURITIBA, BRASIL
}

\section{EVALUATION OF NIGHTIME TRAFFIC NOISE - CASE STUDY IN THE CITY OF CURITIBA, BRAZIL}

\author{
Elaine Carvalho da Paz \\ Companhia de Saneamento do Paraná (SANEPAR) \\ Unidade de Serviços de Obras e Projetos Sudeste - USPO/SD \\ Ponta Grossa,PR \\ e-mail: pazelaine@hotmail.com \\ Paulo Henrique Zannin \\ Professor da Universidade Federal do Paraná (UFPR) \\ Departamento de Mecânica \\ Curitiba, $P R$ \\ e-mail: zannin@ufpr.br
}

Recebido em: 03/02/2013

Aceito em: 20/12/2013

\section{Resumo}

O ruído é um dos mais importantes poluidores ambientais, por isto sua quantificação se faz necessária. As imissões sonoras noturnas geradas por um trecho urbano de uma rodovia brasileira, foram avaliados através de medições in situ. A partir dos dados coletados foi elaborada uma modelagem matemática para alguns descritores acústicos como o nível sonoro equivalente Leq, e para os níveis sonoros estatísticos L10, L50 e L90, em função da correlação destes entre si e com variáveis de tráfego, para cálculo de níveis sonoros no período noturno. As variáveis de trafego utilizadas foram: número de veículos leves (LV); número de veículos pesados (HV); número total de veículos (TV); fluxo de veículos leves por hora (LF); fluxo de veículos pesados por hora (HF); fluxo total de veículos por hora (TF); porcentagem de veículos pesados (PHV); e porcentagem de veículos leves (PLV). Os modelos desenvolvidos apresentam três características: a) são para aplicação ao ruído de tráfego noturno, b) para a aplicação de regressão linear e não logarítmica, c) e para a aplicação de intervalos de classe para descrever a complexidade da faixa dinâmica de imissões sonoras, desde os níveis mais baixos até os mais elevados. Verificou-se que para amostras grandes $(n>30)$ e predição do valor do nível sonoro equivalente (Leq), a avaliação com intervalos de classe de ruído é a mais indicada. 
Palavras-chave: poluição sonora, ruído de tráfego, período noturno, modelo de predição.

\section{Abstract}

Noise is one of the most important environmental polluters, therefore, their quantification is needed. The night immission noise levels generated by a urban stretch of highway in Brazil were evaluated by in situ measurements. From the collected data was elaborated a mathematical modeling of the acoustic descriptors as the equivalent sound level Leq and statistical noise levels L10, L50 and L90, depending on the correlation of these variables with each other and with traffic, to calculate levels sound, at nighttime. The following variables of traffic were used: number of light vehicles (LV); number of heavy vehicles (HV); total number of vehicles (TV); flow of light vehicles per hour (LF); flow of heavy vehicles per hour (HF); total flow of vehicles per hour (TF); percentage of heavy vehicles (PHV); and percentage of light vehicles (PLV). The developed models have three potential applications/characteristics: a) traffic noise at night, b) linear e not logarithmic regression, c) class intervals to describe the complexity of the dynamic range of sound immissions from lowest to highest levels. It was found that for the prediction value of the equivalent noise level (Leq) in large samples $(n>30)$, the prediction of noise levels with class intervals is the most suitable approach.

Keywords: noise pollution, traffic noise, nighttime, prediction model.

\section{INTRODUÇÃO}

O crescimento econômico em todo o mundo traz em seu cerne o crescimento da população e em decorrência disto o crescimento das cidades. Segundo a edição de Janeiro de 2011 da National Geographic (2011), em 1975 existiam somente três cidades no mundo com mais de 10 milhões de habitantes. Hoje temos 21 megacidades com mais de 10 milhões de habitantes. Indubitavelmente, esta crescente população traz consigo entre outros tipos de poluição, a poluição sonora (BELOJEVIC e JAKOVLJEVIC, 1997; ZANNIN et al., 2002; ZANNIN e SZEREMETA, 2003; PAZ et al., 2005; ZANNIN et al., 2006; BELOJEVIC et al., 2008; ZANNIN, 2008; JAKOVLJEVIC et al., 2009, SZEREMETA e ZANNIN, 2009; GUEDES et al., 2011; ZANNIN et al., 2013).

Muitos setores da sociedade são afetados pela poluição sonora, em especial a gerada pelo tráfego de veículos. O ruído de tráfego interfere especialmente e de maneira negativa em atividades onde a atenção e concentração são indispensáveis. Em função do aumento crescente da poluição sonora urbana, estudos da qualidade 
e adequação acústica de ambientes destinados a atividades com grande demanda intelectual e cognitiva, como ambientes educacionais e laborais, tem sido o foco de diversos trabalhos científicos em diversos periódicos acadêmicos (ZANNIN et al., 2003; DÍAZ e PEDRERO, 2006; MUZET, 2007; ZANNIN e MARCON, 2007; ASTOLFI et al., 2008; ZANNIN e ZWIRTES, 2009; ZANNIN e FERREIRA, 2009; PASSERO e ZANNIN, 2009; HAKA et al., 2009; FERNÁNDEZ et al., 2009; PORTELA e ZANNIN, 2010; KLATTE e HELLBRÜCK, 2010; PAZ e ZANNIN, 2012).

O ruído gerado pelo tráfego em rodovias é um problema ambiental, por ser o sistema rodoviário ao longo de suas margens, nos grandes centros urbanos, em especial em países em desenvolvimento e ou subdesenvolvidos, um atrativo à ocupação populacional desordenada, o que contribui para o aumento da poluição sonora urbana devido ao aumento no número de fontes sonoras (MASCHKE, 1999; GERGES, 2004; PAZ e ZANNIN, 2010).

Muitos são os parâmetros de tráfego que apresentam correlação direta ou inversa com a geração dos níveis de ruído de tráfego. Os principais são: o volume de tráfego, a composição do tráfego, a velocidade dos veículos, o gradiente da pista e a distância da fonte ao receptor (SEBERG, 1987; SATTLER, 1999; GARCíA e FAUS, 1991; GOLEBIEWSKI et al., 2003; GOLMOHAMMADI et al., 2007; CALIXTO et al., 2008).

Em relação ao volume de tráfego, o aumento desta parcela, em termos de veículo por hora, causa um aumento gradativo no nível de ruído, até um valor de pico, no qual ocorre a estabilização do sistema para volumes superiores (BURNS, 1973; VDA, 1978; PAZ et al., 2005).

Em relação à composição e a velocidade, para velocidades de 30 a $80 \mathrm{~km} / \mathrm{h}$, o tráfego de veículos pesados gera níveis de ruído superiores aos do tráfego de veículos leves (TO et al., 2002). Assim, a percentagem de veículos pesados deve ser considerada como uma das variáveis determinantes na modelagem do ruído de tráfego. Para velocidades acima de $80 \mathrm{~km} / \mathrm{h}$, esta influência tenderá a ser menor (CALIXTO et al., 2003; TANG e TONG, 2004; PAZ e ZANNIN, 2010).

Nas últimas décadas, têm sido desenvolvidos vários estudos com intuito de formular modelos matemáticos confiáveis para predição dos níveis sonoros, gerados pelo tráfego rodoviário (ALVES FILHO et al., 2004; TANG e TONG, 2004; CALIXTO 
et al., 2003). Estes estudos procuraram correlacionar parâmetros do tráfego rodoviário, como composição e volume de tráfego, com os níveis sonoros, utilizeo técnicas de regressão estatística (PRASCEVIC et al., 1997; CVETKOVIC et al., 1998; SATTLER, 1999; TO et al., 2002). Contudo, a maioria dos modelos são desenvolvidos para análise do ambiente noturno e tendo como base a regressão logarítimica, sem que seja considerada a multiplicidade do modelo (PAZ e ZANNIN, 2010).

Neste sentido, este trabalho visa apresentar uma modelagem matemática para predição do ruído de tráfego urbano, para o período noturno, tendo como base a regressão linear e considerando intervalos de classe para a descrição da faixa dinâmica de flutuação dos níveis sonoros (mais baixos até os mais elevados). Os modelos de predição assim obtidos diferenciam-se de modelos correntes encontrados na literatura, que utilizam regressão logarítmica e não utilizam intervalos de classe de ruído (BURGESS, 1977; DBV, 1990; MAKAREWICZ, 1998; MAKAREWICZ et al., 1999; SUKSAARD et al., 1999; STEELE, 2001; CALIXTO et al., 2003; ALVES FILHO et al., 2004; CALIXTO et al., 2008).

\section{MATERIAIS E MÉTODOS}

Tomando como estudo de caso o trecho urbano da rodovia BR-476, em Curitiba-PR, Brasil, foi elaborada a modelagem matemática para os principais níveis sonoros, a saber, nível sonoro equivalente $\left(L_{e q}\right)$ e níveis sonoros estatísticos $\left(L_{10}\right)$, $\left(L_{50}\right)$ e $\left(L_{90}\right)$. Todos os níveis sonoros foram medidos na curva de ponderação "A" (Figura 1), por ser esta a curva mais representativa da sensação auditiva e, portanto, a mais indicada para o estudo do ruído em comunidade, ruído de tráfego, conforto acústico etc.. As etapas que englobaram a avaliação do ambiente acústico foram: estudo piloto, medições, tabulação dos dados medidos e modelagem matemática. 
Figura 1: Curva de ponderação $A$

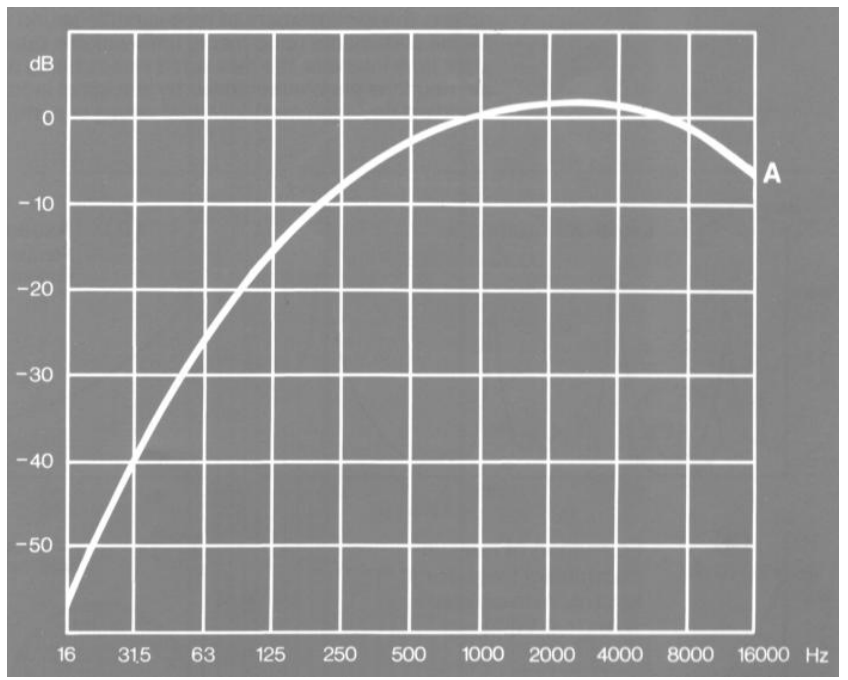

Fonte: V.D.A., 1978

\section{ESTUDO PILOTO}

Nesta etapa, foram avaliados as construtivas da via, a logística de tráfego da via, o planejamento urbano da área e a caracterização sócio-econômica da área de abrangência da via.

Para o controle de variáveis da via em estudo, foram selecionados trechos que apresentavam as seguintes características: trecho retilíneo e plano, sem bruscas variações do gradiente da pista de rodagem; rampas inferiores e/ou iguais a 5\%; rodovia Classe I-A (classificação brasileira); região de entorno em sua grande maioria plana; existência de dispositivos de controle de velocidade e parada de veículos; existência de seções disciplinadoras de tráfego em nível e em desnível; pistas com três faixas, com 3,60 m de largura; existência de via marginal de mão única em quase toda a extensão oeste; acostamento com largura média de 1,50 m; canteiro central com larguras variáveis de $0,50 \mathrm{~m}$ e $20 \mathrm{~m}$; existência de sistema de drenagem eficiente em todo o trecho; pavimento sem defeitos estruturais; revestimento em concreto betuminoso usinado a quente (CBUQ); existência de sinalização vertical e horizontal funcionais e em boas condições; tráfego misto; zona de localização mista, com predominância de estabelecimentos comerciais e com existência de edificações de uso especial, como por exemplo, educacional, e residências de médio a alto padrão; velocidade média fixada para o tráfego de 60 $\mathrm{km} / \mathrm{h}$; velocidade real média verificada de $80 \mathrm{~km} / \mathrm{h}$ para veículos leves e $70 \mathrm{~km} / \mathrm{h}$ 


\section{Avaliação do ruído de tráfego noturno - estudo de caso na cidade de Curitiba, Brasil}

para veículos pesados; tráfego de veículos de transporte coletivo de regiões metropolitanas.

\section{MEDIÇÕES}

Seguindo as características pré-definidas pelo estudo piloto, foram selecionados 24 pontos ao longo da via, em uma extensão de $11,5 \mathrm{~km}$, espaçados entre si em 500 m para realização das medições sonoras (ZANNIN e SANT'ANA, 2011). As medições in situ foram realizadas no período noturno (22:01 h às 7:00 h), em cada ponto durante 15 minutos, conforme recomenda Romeu et al. (2011).

Estas medições foram realizadas seguindo os critérios da norma alemã $R L S$ 90 (DBV, 1990), sendo a aplicação desta norma válida para estudos de vias brasileiras, conforme pesquisas anteriores ao presente trabalho (CALIXTO et. al., 2003; PAZ e ZANNIN, 2010).

A norma alemã indica que as medições sonoras sejam efetuadas a $25 \mathrm{~m}$, perpendicularmente ao eixo da pista e a $15 \mathrm{~m}$, perpendicularmente ao eixo da pista quando da ocorrência de recuos muito próximos de edificações e com altura do aparelho analisador de pressão sonora de 1,20 m. Em 6 pontos 0 aparelho foi posicionado no canteiro central de divisão da via, em função da largura do mesmo e da distância máxima de medição de $25 \mathrm{~m}$ estipulada pela norma RLS-90 (DBV, 1990).

O processo de contagem de veículos foi manual, seguindo as recomendações do DNER (DANTAS, 1996), que caracteriza veículos leves e veículos pesados, com tempo de contagem de 15 minutos.

As medições dos níveis sonoros foram realizadas com a utilização do medidor sonoro modelo BK 2238, da Bruel \& Kjaer, classe I, fixado em um tripé, com microfone tipo 4188, com tempo de medição fixo de 5 minutos, com uma faixa dinâmica de 40 a 120 dB, na curva de ponderação "A".

Cabe ressaltar que antes de cada seção de medições e após esta, o medidor sonoro foi calibrado, utilizando-se o calibrador tipo 4231 da Brüel \& Kjaer.

Todas as medições foram realizadas em boas condições meteorológicas (sem chuva e vento forte), em trechos retilíneos, distante em no mínimo $50 \mathrm{~m}$ de dispositivos de redução e controle de velocidade de veículos. 
Os dados coletados foram: número de veículos leves e pesados; nível sonoro equivalente na curva de ponderação " $A$ " $\left(L_{e q}\right)$; níveis sonoros estatísticos $\left(L_{10}\right),\left(L_{50}\right)$ e $\left(L_{90}\right)$; níveis sonoros, máximo e mínimo $\left(L_{m a ́ x}, L_{m i ́ n}\right)$. Ressalta-se que a unidade de medição dos níveis sonoros foi o $\mathrm{dB}(\mathrm{A})$, a saber, decibéis na curva de ponderação "A".

\section{DEFINIÇÕES}

Para entendimento do texto, apresenta-se uma breve definição sobre os níveis sonoros estatísticos. Os níveis sonoros estatísticos são níveis de pressão sonora que são ultrapassados durante uma determinada fração do tempo total de medição. Os níveis estatísticos de maior interesse para estudos de ruído de tráfego são $\left(L_{10}\right),\left(L_{50}\right)$ e $\left(L_{90}\right)$, que são os níveis excedidos durante, respectivamente, $10 \%$, $50 \%$ e $90 \%$ do tempo de medição. Para o estudo de ruído de tráfego rodoviário, o nível estatístico $L_{10}$ pode ser aceito aproximadamente como valores de pico, pois ele indica valores que foram excedidos durante apenas $10 \%$ do tempo total de medição. Já o nível estatístico $L_{90}$, pode ser aceito como sendo um ruído de fundo, posto que ele indica o nível de ruído que foi ultrapassado durante quase todo o tempo de medição (MAUTE, 2006).

\section{TABULAÇÃO DOS DADOS MEDIDOS}

Com os dados obtidos pela contagem de tráfego foram determinados a demanda média de tráfego efetiva, o fluxo efetivo noturno e as composições percentuais efetivas do tráfego.

Os dados de medição dos níveis sonoros foram classificados e submetidos à análise do software EVALUATOR BK 7820, pelo qual foram obtidos os valores médios para os níveis sonoros equivalentes $\left(L_{e q}\right)$, os níveis sonoros estatísticos $\left(L_{10}\right),\left(L_{50}\right)$ e $\left(L_{90}\right)$, e os níveis sonoros, máximo e mínimo $\left(L_{m a ́ x}, L_{m i n}\right)$ para o período noturno, de cada ponto.

\section{MODELOS MATEMÁTICOS PARA A AVALIAÇÃO DO RUÍDO DE TRÁFEGO NOTURNO}

As técnicas utilizadas para a modelagem matemática foram a regressão linear simples e múltipla. Para validação dos modelos desenvolvidos, foram 
realizados os seguintes testes estatísticos: medidas dos graus de associação entre membros da equação (coeficiente de correlação linear "R" e coeficiente de

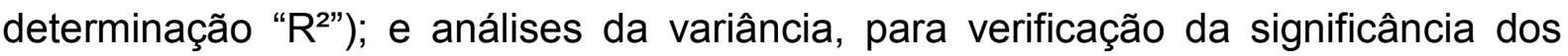
regressores.

As análises de regressão linear efetuadas consideraram a modelagem matemática em duas situações: sem intervalos de classe, ou seja, sem restrição da faixa de atuação da variável independente; e com intervalos de classe, ou seja, com restrição da faixa de atuação da variável independente, a partir do ponto de média dos dados amostrais das variáveis dependentes.

A amostragem gerou dois grupos de dados, um contento os valores medidos e outro contendo os valores médios calculados. Da análise estatística descritiva dos grupos foram calculadas as suas medidas de posição e dispersão. Em seguida, foi efetuado o cálculo da matriz de correlação, para a determinação de quais variáveis deveriam ser utilizadas na elaboração do modelo matemático, em função do seu grau de correlação com os níveis sonoros principais.

Os resultados foram submetidos ao teste de normalidade de Shapiro Wilk. Da análise do teste de normalidade, foi identificada que a distribuição dos dados coletados e calculados obedecia aproximadamente à uma distribuição normal. Da a análise gráfica BOX-PLOT, foi avaliada a simetria dos dados e sua dispersão, sendo comparadas todas as variáveis consideradas para o estudo.

Para tratamento dos dados e análises estatísticas foram utilizados os softwares MATLAB Release 12, STATISTICA 5.1 e ACTION+R 2.5.1.

As variáveis consideradas para o estudo foram: nível de pressão sonora equivalente $\left(L_{e q}\right)$; níveis estatísticos de pressão sonora $\left(L_{10}\right),\left(L_{50}\right)$ e $\left(L_{90}\right)$; nível de pressão sonora máximo $\left(L_{\text {máx }}\right)$; nível de pressão sonora mínimo $\left(L_{\text {mín }}\right)$; número de veículos leves (LV); número de veículos pesados (HV); número total de veículos (TV); fluxo de veículos leves por hora (LF); fluxo de veículos pesados por hora (HF); fluxo total de veículos por hora (TF); porcentagem de veículos pesados (PHV); e porcentagem de veículos leves (PLV). 


\section{RESULTADOS E DISCUSSÃO}

A análise gráfica BOX-PLOT, que apresenta os valores de medidas de posição e quartis (Figuras 2 a 5), demonstrou não haver diferença de tendência entre as características das amostras medidas para as amostras de valores médios de cada ponto, o que qualificou ambas as amostras à modelagem matemática.

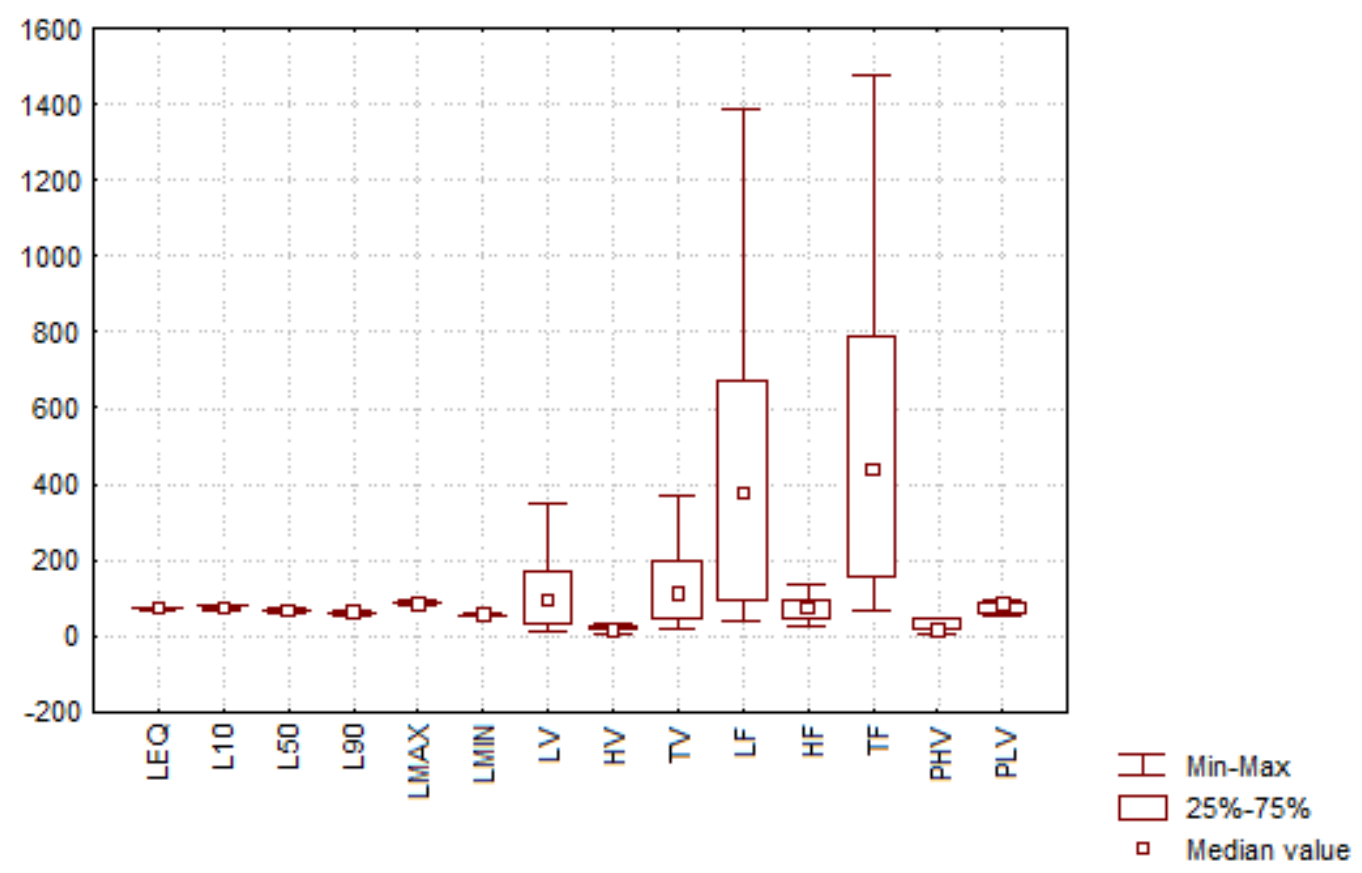

Figura 2: Box-plot (mínimo e máximo, quartis, mediana), amostra medida/noturno.

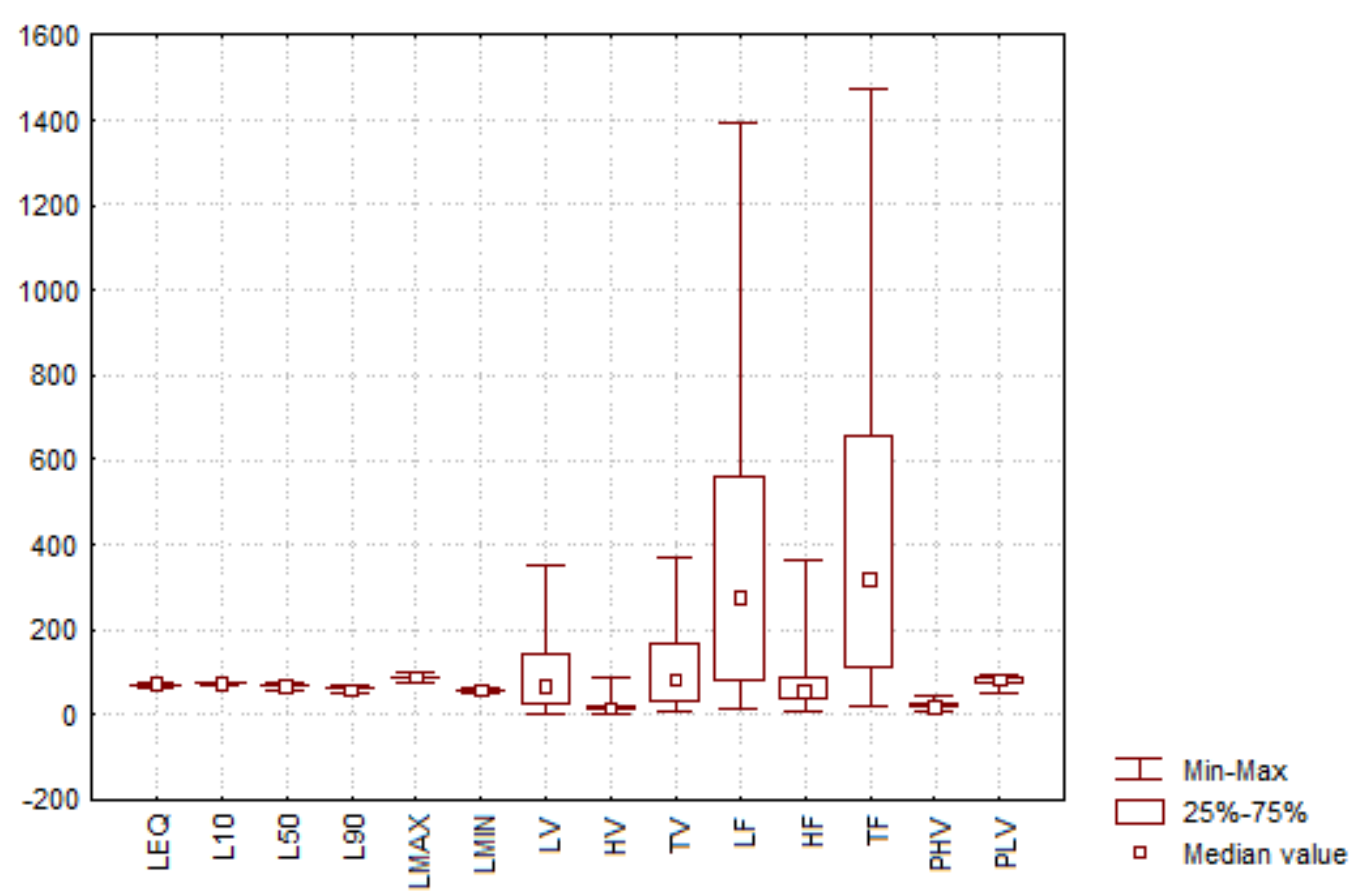

Figura 3: Box-plot (mínimo e máximo, quartis, mediana), amostra média/noturno. 


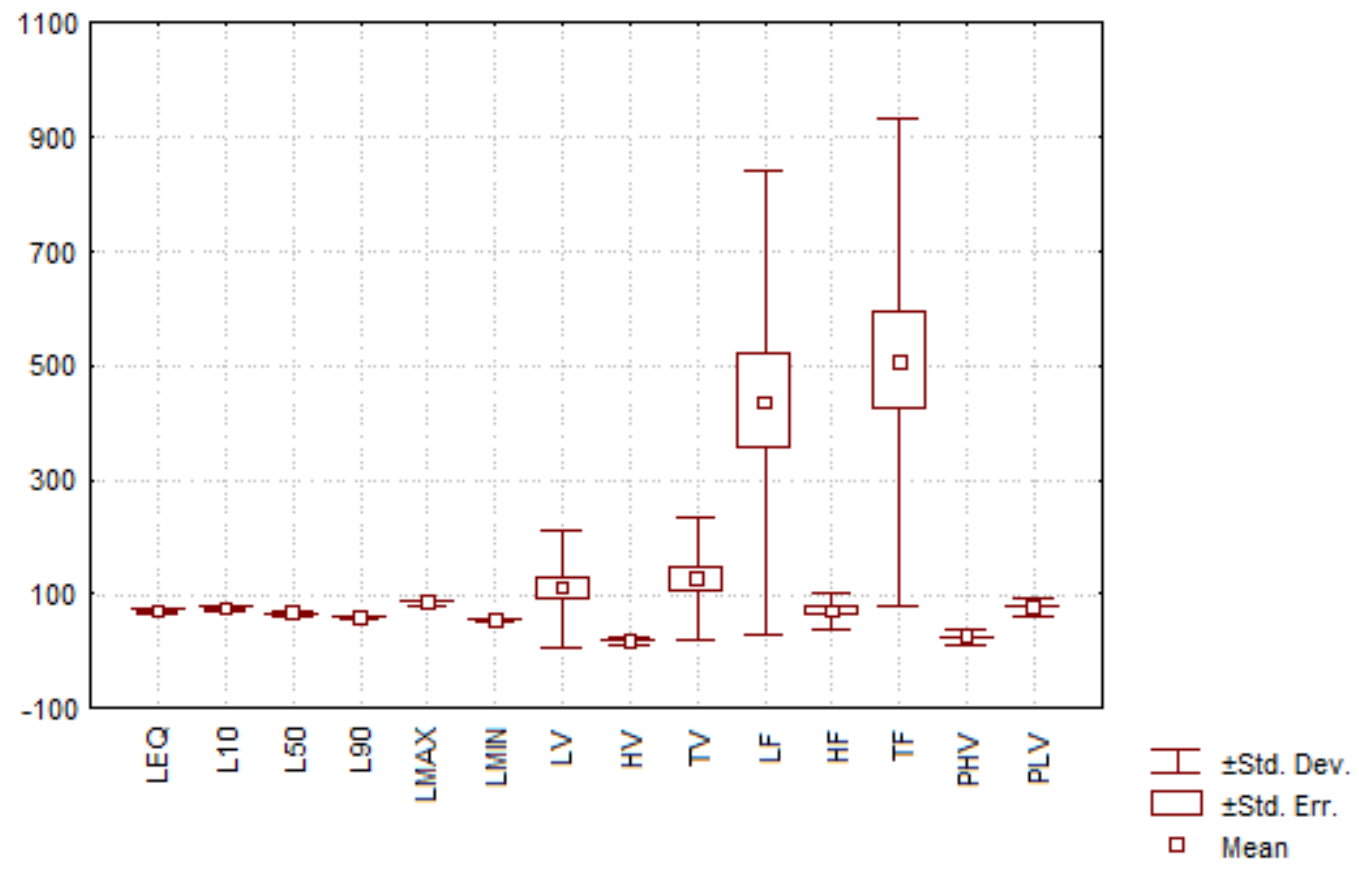

Figura 4: Box-plot (desvio padrão, erro padrão, média), amostra medida/noturno.

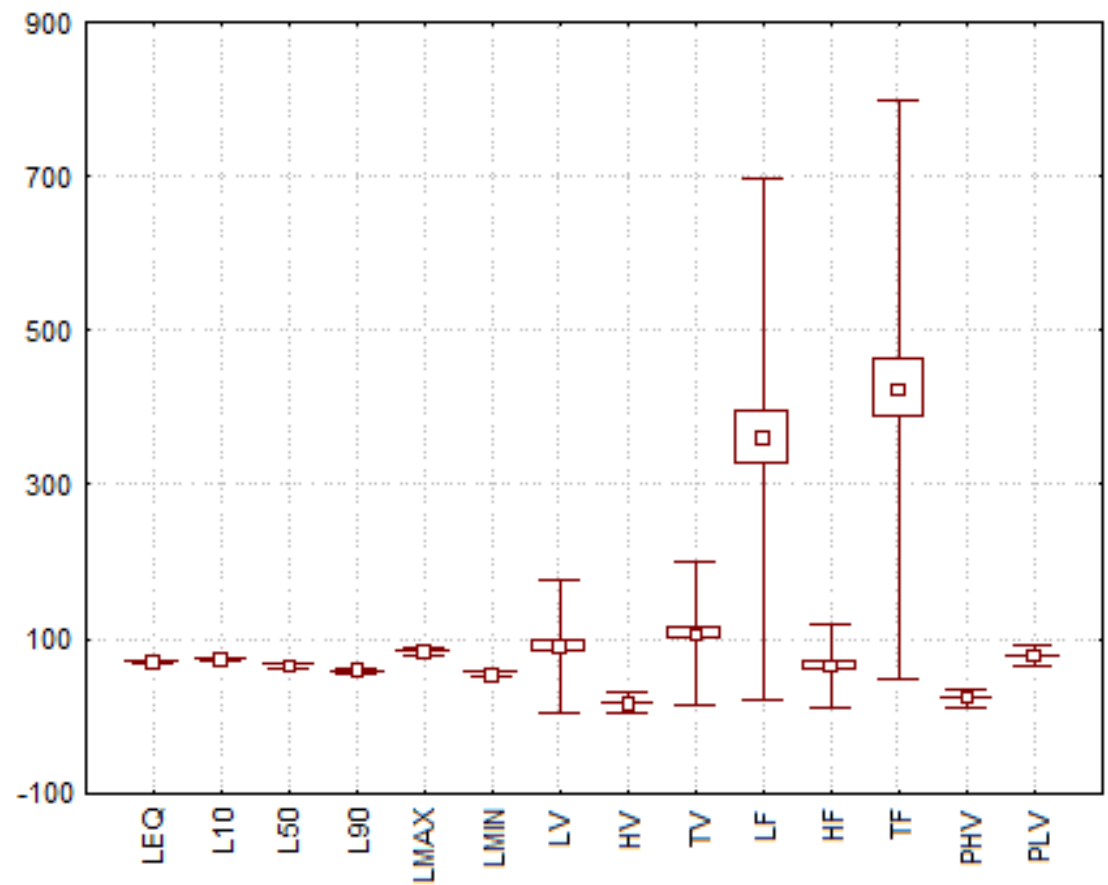

Figura 5: Box-plot (desvio padrão, erro padrão, média), amostra média/noturno.

As Tabelas 1 e 2, a seguir, apresentam os resultados da análise de $B O X-$ PLOT para os níveis sonoros e variáveis de tráfego. 
TABELA 1 - RESULTADOS DA ANÁLISE BOX-PLOT PARA NÍVEIS SONOROS (MÍNIMO E MÁXIMO, MÉDIA, MEDIANA)

\begin{tabular}{|c|c|c|c|c|}
\hline \multirow{2}{*}{$\begin{array}{c}\text { Níveis } \\
\text { Sonoros }\end{array}$} & \multicolumn{4}{|c|}{ Amostra medida } \\
\hline & Mínimo & Máximo & Média & Mediana \\
\hline $\mathrm{L}_{\mathrm{eq}}[\mathrm{dB}(\mathrm{A})]$ & 63,40 & 75,80 & 69,02 & 69,25 \\
\hline $\mathrm{L}_{10}[\mathrm{~dB}(\mathrm{~A})]$ & 66,50 & 76,40 & 72,56 & 73,60 \\
\hline $\mathrm{L}_{50}[\mathrm{~dB}(\mathrm{~A})]$ & 58,90 & 72,10 & 64,89 & 65,50 \\
\hline $\mathrm{L}_{90}[\mathrm{~dB}(\mathrm{~A})]$ & 51,10 & 67,90 & 57,97 & 58,85 \\
\hline $\mathrm{L}_{\operatorname{MAX}}[\mathrm{dB}(\mathrm{A})]$ & 76,70 & 102,20 & 84,09 & 84,40 \\
\hline $\mathrm{L}_{\mathrm{MIN}}[\mathrm{dB}(\mathrm{A})]$ & 48,10 & 64,70 & 53,68 & 54,20 \\
\hline Níveis & \multicolumn{4}{|c|}{ Amostra média } \\
\hline Sonoros & Mínimo & Máximo & Média & Mediana \\
\hline $\mathrm{L}_{\mathrm{eq}}[\mathrm{dB}(\mathrm{A})]$ & 64,20 & 72,90 & 69,07 & 69,25 \\
\hline $\mathrm{L}_{10}[\mathrm{~dB}(\mathrm{~A})]$ & 67,30 & 75,40 & 72,63 & 72,89 \\
\hline $\mathrm{L}_{50}[\mathrm{~dB}(\mathrm{~A})]$ & 60,20 & 71,60 & 64,85 & 65,10 \\
\hline $\mathrm{L}_{90}[\mathrm{~dB}(\mathrm{~A})]$ & 52,70 & 66,80 & 57,95 & 58,80 \\
\hline$L_{\operatorname{MAX}}[\mathrm{dB}(\mathrm{A})]$ & 79,00 & 93,90 & 84,22 & 83,90 \\
\hline $\mathrm{L}_{\mathrm{MIN}}[\mathrm{dB}(\mathrm{A})]$ & 49,70 & 61,90 & 53,66 & 53,90 \\
\hline
\end{tabular}

Legenda: nível de pressão sonora equivalente $\left(L_{e q}\right)$; níveis estatísticos de pressão sonora $\left(L_{10}\right),\left(L_{50}\right)$ e

$\left(L_{90}\right)$; nível de pressão sonora máximo $\left(L_{m a ́ x}\right)$; nível de pressão sonora mínimo $\left(L_{\text {mín }}\right)$.

TABELA 2 - RESULTADOS DA ANÁLISE BOX-PLOT PARA VARIÁVEIS DE TRÁFEGO (MÍNIMO E MÁXIMO, MÉDIA, MEDIANA)

\begin{tabular}{|c|c|c|c|c|}
\hline \multirow{2}{*}{$\begin{array}{c}\text { Variáveis de } \\
\text { Tráfego }\end{array}$} & \multicolumn{4}{|c|}{ Amostra medida } \\
\hline & Mínimo & Máximo & Média & Mediana \\
\hline LV [veículo] & 3 & 348 & 90 & 68 \\
\hline HV [veículo] & 2 & 90 & 16 & 13 \\
\hline TV [veículo] & 5 & 369 & 106 & 80 \\
\hline LF [veículo/h] & 12 & 1392 & 359 & 272 \\
\hline HF [veículo/h] & 8 & 360 & 92 & 52 \\
\hline TF [veículo/h] & 20 & 1476 & 424 & 318 \\
\hline PHV [\%] & 5,69 & 46,67 & 22,28 & 17,83 \\
\hline PLV [\%] & 53,33 & 94,31 & 77,72 & 82,17 \\
\hline \multirow{2}{*}{$\begin{array}{l}\text { Variáveis de } \\
\text { Tráfego }\end{array}$} & \multicolumn{4}{|c|}{ Amostra média } \\
\hline & Mínimo & Máximo & Média & Mediana \\
\hline LV & 9 & 348 & 109 & 93 \\
\hline $\mathrm{HV}$ & 7 & 33 & 18 & 18 \\
\hline TV & 16 & 369 & 127 & 109 \\
\hline LF & 36 & 1392 & 437 & 374 \\
\hline $\mathrm{HF}$ & 28 & 132 & 71 & 70 \\
\hline TF & 64 & 1476 & 507 & 436 \\
\hline PHV & 5,69 & 46,67 & 24,32 & 16,64 \\
\hline PLV & 53,33 & 94,31 & 75,68 & 83,37 \\
\hline
\end{tabular}

Legenda:número de veículos leves (LV); número de veículos pesados (HV); número total de veículos (TV); fluxo de veículos leves por hora (LF); fluxo de veículos pesados por hora (HF); fluxo total de veículos por hora (TF); porcentagem de veículos pesados (PHV); e porcentagem de veículos leves (PLV).

As Tabelas 3 e 4, a seguir, apresentam os resultados de correlação de variáveis: 


\section{TABELA 3 - MATRIZ DE CORRELAÇÃO PARA A AMOSTRA MÉDIA/NOTURNO}

\begin{tabular}{|c|c|c|c|c|c|c|c|c|c|c|c|c|c|c|}
\hline & $\mathrm{L}_{\mathrm{eq}}$ & $\mathrm{L}_{10}$ & $L_{50}$ & $L_{90}$ & $\mathrm{~L}_{\mathrm{MAX}}$ & $L_{\text {MIN }}$ & LV & $\mathrm{HV}$ & TV & LF & $\mathrm{HF}$ & $\mathrm{TF}$ & PHV & PLV \\
\hline $\mathrm{L}_{\mathrm{eq}}$ & 1,00 & 0,97 & 0,85 & 0,74 & 0,73 & 0,70 & 0,63 & 0,73 & 0,66 & 0,63 & 0,73 & 0,66 & $-0,40$ & 0,36 \\
\hline $\mathrm{L}_{10}$ & 0,97 & 1,00 & 0,76 & 0,63 & 0,71 & 0,60 & 0,46 & 0,66 & 0,49 & 0,46 & 0,66 & 0,49 & $-0,18$ & 0,18 \\
\hline $\mathrm{L}_{50}$ & 0,85 & 0,76 & 1,00 & 0,95 & 0,35 & 0,89 & 0,83 & 0,86 & 0,86 & 0,83 & 0,86 & 0,86 & $-0,74$ & 0,74 \\
\hline $\mathrm{L}_{90}$ & 0,74 & 0,63 & 0,95 & 1,00 & 0,26 & 0,97 & 0,76 & 0,84 & 0,79 & 0,76 & 0,84 & 0,79 & $-0,76$ & 0,76 \\
\hline $\mathrm{L}_{\text {MAX }}$ & 0,73 & 0,71 & 0,35 & 0,26 & 1,00 & 0,25 & 0,27 & 0,23 & 0,28 & 0,27 & 0,23 & 0,28 & 0,03 & $-0,03$ \\
\hline $\mathrm{L}_{\mathrm{MIN}}$ & 0,70 & 0,60 & 0,89 & 0,97 & 0,25 & 1,00 & 0,70 & 0,76 & 0,72 & 0,70 & 0,76 & 0,72 & $-0,71$ & 0,71 \\
\hline $\mathrm{LV}$ & 0,63 & 0,46 & 0,83 & 0,76 & 0,27 & 0,70 & 1,00 & 0,65 & 1,00 & 1,00 & 0,65 & 1,00 & $-0,84$ & 0,84 \\
\hline $\mathrm{HV}$ & 0,73 & 0,66 & 0,86 & 0,84 & 0,23 & 0,76 & 0,65 & 1,00 & 0,70 & 0,65 & 1,00 & 0,70 & $-0,56$ & 0,56 \\
\hline TV & 0,66 & 0,49 & 0,86 & 0,79 & 0,28 & 0,72 & 1,00 & 0,70 & 1,00 & 1,00 & 0,70 & 1,00 & $-0,84$ & 0,84 \\
\hline LF & 0,63 & 0,46 & 0,83 & 0,76 & 0,27 & 0,70 & 1,00 & 0,65 & 1,00 & 1,00 & 0,65 & 1,00 & $-0,84$ & 0,84 \\
\hline $\mathrm{HF}$ & 0,73 & 0,66 & 0,86 & 0,84 & 0,23 & 0,76 & 0,65 & 1,00 & 0,70 & 0,65 & 1,00 & 0,70 & $-0,56$ & 0,56 \\
\hline TF & 0,66 & 0,49 & 0,86 & 0,79 & 0,28 & 0,72 & 1,00 & 0,70 & 1,00 & 1,00 & 0,70 & 1,00 & $-0,84$ & 0,84 \\
\hline PHV & $-0,40$ & $-0,18$ & $-0,74$ & $-0,76$ & 0,03 & $-0,71$ & $-0,84$ & $-0,56$ & $-0,84$ & $-0,84$ & $-0,56$ & $-0,84$ & 1,00 & $-1,00$ \\
\hline PLV & 0,36 & 0,18 & 0,74 & 0,76 & $-0,03$ & 0,71 & 0,84 & 0,56 & 0,84 & 0,84 & 0,56 & 0,84 & $-1,00$ & 1,00 \\
\hline & & & & & & & & & & & & & & \\
\hline
\end{tabular}

TABELA 4 - MATRIZ DE CORRELAÇÃO PARA A AMOSTRA MEDIDA/NOTURNO

\begin{tabular}{c|c|c|c|c|c|c|c|c|c|c|c|c|c|c}
\hline & $\mathrm{L}_{\mathrm{eq}}$ & $\mathrm{L}_{10}$ & $\mathrm{~L}_{50}$ & $\mathrm{~L}_{90}$ & $\mathrm{~L}_{\mathrm{MAX}}$ & $\mathrm{L}_{\mathrm{MIN}}$ & $\mathrm{LV}$ & $\mathrm{HV}$ & $\mathrm{TV}$ & $\mathrm{LF}$ & $\mathrm{HF}$ & $\mathrm{TF}$ & $\mathrm{PHV}$ & $\mathrm{PLV}$ \\
\hline $\mathrm{L}_{\mathrm{eq}}$ & 1,00 & 0,96 & 0,83 & 0,74 & 0,74 & 0,72 & 0,50 & 0,51 & 0,51 & 0,50 & 0,51 & 0,51 & $-0,46$ & 0,26 \\
\hline $\mathrm{L}_{10}$ & 0,96 & 1,00 & 0,75 & 0,64 & 0,67 & 0,63 & 0,36 & 0,36 & 0,38 & 0,36 & 0,36 & 0,38 & $-0,13$ & 0,13 \\
\hline $\mathrm{L}_{50}$ & 0,83 & 0,75 & 1,00 & 0,94 & 0,37 & 0,86 & 0,72 & 0,58 & 0,73 & 072 & 0,58 & 0,73 & $-0,58$ & 0,58 \\
\hline $\mathrm{L}_{90}$ & 0,74 & 0,64 & 0,94 & 1,00 & 0,29 & 0,94 & 0,67 & 0,61 & 0,69 & 0,67 & 0,61 & 0,69 & $-0,58$ & 0,58 \\
\hline $\mathrm{L}_{\text {MAX }}$ & 0,74 & 0,67 & 0,37 & 0,29 & 1,00 & 0,36 & 0,18 & 0,07 & 0,17 & 0,18 & 0,07 & 0,17 & $-0,01$ & 0,01 \\
\hline $\mathrm{L}_{\text {MIN }}$ & 0,72 & 0,63 & 0,86 & 0,94 & 0,36 & 1,00 & 0,59 & 0,57 & 0,61 & 0,59 & 0,57 & 0,61 & $-0,48$ & 0,48 \\
\hline LV & 0,50 & 0,36 & 0,72 & 0,67 & 0,18 & 0,59 & 1,00 & 0,60 & 0,99 & 1,00 & 0,60 & 0,99 & $-0,68$ & 0,68 \\
\hline HV & 0,51 & 0,36 & 0,58 & 0,61 & 0,07 & 0,57 & 0,60 & 1,00 & 0,69 & 0,60 & 1,00 & 0,69 & $-0,17$ & 0,17 \\
\hline TV & 0,51 & 0,38 & 0,73 & 0,69 & 0,17 & 0,61 & 0,99 & 0,69 & 1,00 & 0,99 & 0,69 & 1,00 & $-0,64$ & 0,64 \\
\hline LF & 0,50 & 0,36 & 0,72 & 0,67 & 0,18 & 0,59 & 1,00 & 0,60 & 0,99 & 1,00 & 0,60 & 0,99 & $-0,68$ & 0,68 \\
\hline HF & 0,51 & 0,36 & 0,58 & 0,61 & 0,07 & 0,57 & 0,60 & 1,00 & 0,69 & 0,60 & 1,00 & 0,69 & $-0,17$ & 0,17 \\
\hline TF & 0,51 & 0,38 & 0,73 & 0,69 & 0,17 & 0,61 & 0,99 & 0,69 & 1,00 & 0,99 & 0,69 & 1,00 & $-0,64$ & 0,64 \\
\hline PHV & $-0,46$ & $-0,13$ & $-0,58$ & $-0,58$ & $-0,01$ & $-0,48$ & $-0,68$ & $-0,17$ & $-0,64$ & $-0,68$ & $-0,17$ & $-0,64$ & 1,00 & $-1,00$ \\
\hline PLV & 0,26 & 0,13 & 0,58 & 0,58 & 0,01 & 0,48 & 0,68 & 0,17 & 0,64 & 0,68 & 0,17 & 0,64 & $-1,00$ & 1,00 \\
\hline
\end{tabular}

Legenda: Nível de pressão sonora equivalente $\left(L_{e q}\right)$; níveis estatísticos de pressão sonora $\left(L_{10}\right),\left(L_{50}\right)$ e $\left(L_{90}\right)$; nível de pressão sonora máximo $\left(L_{m a ́ x}\right)$; nível de pressão sonora mínimo $\left(L_{\text {mín }}\right)$; número de veículos leves (LV); número de veículos pesados (HV); número total de veículos (TV); fluxo de veículos leves por hora (LF); fluxo de veículos pesados por hora (HF); fluxo total de veículos por hora (TF); porcentagem de veículos pesados (PHV); e porcentagem de veículos leves (PLV). 
Avaliação do ruído de tráfego noturno - estudo de caso na cidade de Curitiba, Brasil

Para os níveis sonoros, verificou-se em ambos os agrupamentos maiores correlações de $\left(L_{e q}\right) \operatorname{com}\left(L_{10}\right),\left(L_{50}\right)$ e $\left(L_{90}\right)$. Já para a correlação entre a variável $L_{e q}$ e as variáveis de tráfego, as maiores correlações verificadas foram com as variáveis HF (fluxo de veículos pesados), TF (fluxo total de veículos) e PHV (percentagem de veículos pesados).

A Tabela 5 apresenta o resumo da análise de correlação para as variáveis selecionadas para a modelagem matemática.

TABELA 5 - RESUMO DA ANÁLISE DE CORRELAÇÃO LINEAR

\begin{tabular}{|c|c|c|c|c|}
\hline $\begin{array}{l}\text { Medições } \\
\text { Período } \\
\text { Noturno } \\
\end{array}$ & Variáveis & $\begin{array}{c}\text { Coeficiente } \\
\text { de } \\
\text { Correlação de Pearson }\end{array}$ & Tipo de Correlação & Grau de Correlação \\
\hline \multirow{6}{*}{$\mathrm{L}_{\mathrm{eq}}$} & $\mathrm{L}_{10}$ & 0,96 & Direta & Muito forte \\
\hline & $\mathrm{L}_{50}$ & 0,83 & Direta & Muito forte \\
\hline & $\mathrm{L}_{90}$ & 0,74 & Direta & Forte \\
\hline & $\mathrm{HF}$ & 0,51 & Direta & Intermediária \\
\hline & TF & 0,51 & Direta & Intermediária \\
\hline & PHV & $-0,46$ & Inversa & Intermediária \\
\hline $\begin{array}{l}\text { Média } \\
\text { Período } \\
\text { Noturno } \\
\end{array}$ & Variáveis & $\begin{array}{c}\text { Coeficiente } \\
\text { de } \\
\text { Correlação de Pearson }\end{array}$ & Tipo de Correlação & Grau de Correlação \\
\hline \multirow{6}{*}{$\mathrm{L}_{\mathrm{eq}}$} & $\mathrm{L}_{10}$ & 0,97 & Direta & Muito forte \\
\hline & $\mathrm{L}_{50}$ & 0,85 & Direta & Forte \\
\hline & $\mathrm{L}_{90}$ & 0,74 & Direta & Forte \\
\hline & $\mathrm{HF}$ & 0,73 & Direta & Forte \\
\hline & TF & 0,66 & Direta & Forte \\
\hline & PHV & 0,40 & Direta & Intermediária \\
\hline
\end{tabular}

As variáveis selecionadas através da análise de correlação de Pearson, para a modelagem matemática, foram submetidas ao teste de normalidade de Shapiro Wilk, a fim de que se verificar se a modelagem poderia ser efetuada por regressão linear (Tabela 6). 
TABELA 6 - RESUMO DA ANÁLISE DE NORMALIDADE DAS VARIÁVEIS SELECIONADAS

\begin{tabular}{c|c|c}
\hline \multirow{2}{*}{ Variável } & \multicolumn{2}{|c}{ p-valor de Shapiro Wilk * } \\
\cline { 2 - 3 } & $\begin{array}{c}\text { para amostras de } \\
\text { valores medidos }\end{array}$ & $\begin{array}{c}\text { para amostras de } \\
\text { valores médios }\end{array}$ \\
\hline $\mathrm{L}_{\mathrm{eq}}$ & 0,124430037608953 & 0,346372211732969 \\
\hline $\mathrm{L}_{10}$ & 0,132444674082646 & 0,426013502561087 \\
\hline $\mathrm{L}_{50}$ & 0,227520699958085 & 0,165499376749773 \\
\hline $\mathrm{L}_{90}$ & 0,286766304639562 & 0,989792146849463 \\
\hline $\mathrm{HF}$ & 0,150109487725592 & 0,119460632714127 \\
\hline $\mathrm{TF}$ & 0,394464598274046 & 0,106310465059218 \\
\hline $\mathrm{PHV}$ & 0,394464598274046 & 0,101633920884424 \\
\hline
\end{tabular}

${ }^{*}$ A distribuição da amostra é considerada normal para p-valor $\geq 0,05$.

O teste de normalidade de Shapiro Wilk demonstrou que os dados, para todas as variáveis selecionadas para o modelamento matemático, obedeciam aproximadamente a uma distribuição normal. Assim, comprovou-se que a regressão linear poderia ser satisfatoriamente aplicada no desenvolvimento da modelagem matemática, sendo "simples", para o modelamento entre níveis sonoros, e "múltipla" para o modelamento entre $\circ L_{e q}$ e as variáveis de tráfego.

A análise de regressão linear efetuada considerou a modelagem matemática em duas situações: sem intervalos de classe e com intervalos de classe. As variáveis $\left(L_{10}\right),\left(L_{50}\right)$ e $\left(L_{90}\right)$ foram denominadas variáveis dependentes e $\left(L_{e q}\right)$ variável independente. A confiabilidade dos modelos matemáticos é de $95 \%$, uma vez que os mesmos foram calculados a um nível de significância de $5 \%$.

As análises de regressão linear simples e múltipla, efetuadas sem intervalos de classes, forneceram os seguintes modelos matemáticos (Tabela 7):

TABELA 7 - MODELOS MATEMÁTICOS SEM INTERVALO DE CLASSE

\begin{tabular}{c|c|c|l}
\hline Modelo & Equação & Tipo amostral & $\begin{array}{c}\text { Particionamento } \\
\text { amostral }\end{array}$ \\
\hline 1 & $L_{10}=\left(0,889 L_{e q}\right)+11,247$ & valores médios & $\mathrm{s} /$ intervalo de classe \\
\hline 2 & $L_{10}=\left(0,903 L_{e q}\right)+10,004$ & valores medidos & $\mathrm{s} /$ intervalo de classe \\
\hline 3 & $L_{50}=\left(1,323 L_{e q}\right)-26,508$ & valores médios & $\mathrm{s} /$ intervalo de classe \\
\hline 4 & $L_{50}=\left(1,168 L_{e q}\right)-15,704$ & valores medidos & $\mathrm{s} /$ intervalo de classe \\
\hline 5 & $L_{90}=\left(1,223 L_{e q}\right)-26,508$ & valores médios & $\mathrm{s} /$ intervalo de classe \\
\hline 6 & $L_{90}=\left(1,092 L_{e q}\right)-17,402$ & valores medidos & $\mathrm{s} /$ intervalo de classe \\
\hline 7 & $L_{e q}=(0,006 T F)+(0,099 P H V)+63,312$ & valores médios & $\mathrm{s} /$ intervalo de classe \\
\hline 8 & $L_{e q}=(0,039 H F)+(0,001 T F)+65,493$ & valores médios & $\mathrm{s} /$ intervalo de classe \\
\hline 9 & $L_{e q}=(0,004 T F)+(0,027 P H V)+66,630$ & valores medidos & $\mathrm{s} /$ intervalo de classe \\
\hline 10 & $L_{e q}=(0,007 H F)+(0,003 T F)+67,303$ & valores medidos & $\mathrm{s} /$ intervalo de classe \\
\hline
\end{tabular}


Os testes de graus de associação dos modelos demonstraram que para os modelos 1 a 8 , as variáveis das equações apresentam graus de correlação $(R)$ satisfatórios; os graus de determinação $\left(R^{2}\right)$ calculados demonstraram que existe uma proporção de variação total satisfatória que explica a variável dependente. Os graus de associação calculados para os modelos são apresentados na Tabela 8 , a seguir.

TABELA 8 - GRAUS DE ASSOCIAÇÃO, MODELOS DE 1 A 10

\begin{tabular}{c|c|c|c|c}
\hline Modelo & $\mathbf{R 1}$ & Grau de R1 & $\mathbf{R 1}^{2}$ & Grau de R12 $^{\mathbf{2}}$ \\
\hline 1 & 0,98 & Muito forte & 0,94 & Muito forte \\
\hline 2 & 0,96 & Muito forte & 0,92 & Muito forte \\
\hline 3 & 0,85 & Forte & 0,72 & Forte \\
\hline 4 & 0,83 & Forte & 0,69 & Forte \\
\hline 5 & 0,74 & Forte & 0,54 & Intermediária \\
\hline 6 & 0,73 & Forte & 0,53 & Intermediária \\
\hline Modelo & $\mathbf{R 2}$ & Grau de R2 & $\mathbf{R 2}^{2}$ & Grau de R2 $^{\mathbf{2}}$ \\
\hline 7 & 0,75 & Forte & 0,56 & Intermediária \\
\hline 8 & 0,76 & Forte & 0,58 & Intermediária \\
\hline 9 & 0,52 & Intermediária & 0,27 & Fraca \\
\hline 10 & 0,52 & Intermediária & 0,27 & Fraca \\
\hline
\end{tabular}

Legenda: Coeficiente de correlação linear simples (R1); coeficiente de correlação linear múltipla (R2); coeficiente de determinação linear simples $\left(R 1^{2}\right)$; coeficiente de determinação linear múltipla $\left(R 2^{2}\right)$.

Os modelos 9 e 10 não apresentaram graus de associação satisfatórios. Isto se deve ao fato de que a amostra de valores medidos apresenta uma variação maior em suas medidas de dispersão em relação à amostra de valores médios, uma vez que, esta última, em função do cálculo do valor médio, sofre um ajustamento dos dados. Em função destes resultados, as variáveis foram novamente submetidas à análise de regressão, porém, com intervalos de classe, com divisão de cada amostra em duas classes no ponto de média da variável dependente.

A seguir, são apresentados os modelos matemáticos calculados com intervalos de classe, ao nível de significância de 5\% (Tabela 9): 
Avaliação do ruído de tráfego noturno - estudo de caso na cidade de Curitiba, Brasil

TABELA 9 - MODELOS MATEMÁTICOS COM INTERVALO DE CLASSE

\begin{tabular}{|c|c|c|c|}
\hline Modelo & Equação & Tipo amostral & $\begin{array}{c}\text { Particionamento } \\
\text { amostral }\end{array}$ \\
\hline 11 & $\begin{array}{c}L_{10}=\left(1,053 L_{e q}\right)+0,151 \\
L_{10}=\left(0,529 L_{e q}\right)+36,787\end{array}$ & valores médios & $\begin{array}{l}L_{10} \leq 72,62 \\
L_{10}>72,62\end{array}$ \\
\hline 12 & $\begin{array}{c}L_{10}=\left(1,037 L_{e q}\right)+1,064 \\
L_{10}=\left(0,497 L_{e q}\right)+39,092\end{array}$ & valores medidos & $\begin{array}{l}L_{10} \leq 72,56 \\
L_{10}>72,56\end{array}$ \\
\hline 13 & $\begin{array}{l}L_{50}=\left(0,632 L_{e q}\right)+18,817 \\
L_{50}=\left(0,779 L_{e q}\right)+12,838\end{array}$ & valores médios & $\begin{array}{l}L_{50} \leq 64,85 \\
L_{50}>64,85\end{array}$ \\
\hline 14 & $\begin{array}{l}L_{50}=\left(0,595 L_{e q}\right)+21,507 \\
L_{50}=\left(0,561 L_{e q}\right)+28,352\end{array}$ & valores medidos & $\begin{array}{l}L_{50} \leq 64,89 \\
L_{50}>64,89\end{array}$ \\
\hline 15 & $\begin{array}{l}L_{90}=\left(0,456 L_{e q}\right)+23,684 \\
L_{90}=\left(0,512 L_{e q}\right)+24,816\end{array}$ & valores médios & $\begin{array}{l}L_{90} \leq 57,95 \\
L_{90}>57,95\end{array}$ \\
\hline 16 & $\begin{array}{l}L_{90}=\left(0,508 L_{e q}\right)+20,159 \\
L_{90}=\left(0,433 L_{e q}\right)+30,478\end{array}$ & valores medidos & $\begin{array}{l}L_{90} \leq 57,97 \\
L_{90}>57,97\end{array}$ \\
\hline 17 & $\begin{array}{l}L_{e q}=(0,007 T F)+(0,140 P H V+60,731 \\
L_{e q}=(0,003 T F)+(0,076 P H V)+66,971\end{array}$ & valores médios & $\begin{array}{l}L_{e q} \leq 69,07 \\
L_{e q}>69,07\end{array}$ \\
\hline 18 & $\begin{array}{l}L_{e q}=(0,068 H F)+(0,009 T F)+65,577 \\
L_{e q}=(0,014 H F)+(0,001 T F)+65,659\end{array}$ & valores médios & $\begin{array}{l}L_{e q} \leq 69,07 \\
L_{e q}>69,07\end{array}$ \\
\hline 19 & $\begin{array}{l}L_{e q}=(0,005 T F)+(0,052 P H V)+63,800 \\
L_{e q}=(0,002 T F)+(0,001 P H V)+69,863\end{array}$ & valores medidos & $\begin{array}{l}\mathrm{L}_{e q} \leq 69,02 \\
\mathrm{~L}_{e q}>69,02\end{array}$ \\
\hline 20 & $\begin{array}{l}L_{e q}=(0,048 H F)+(0,002 T F)+64,613 \\
L_{e q}=(0,004 H F)+(0,001 T F)+69,807\end{array}$ & valores medidos & $\begin{array}{l}L_{e q} \leq 69,02 \\
L_{e q}>69,02\end{array}$ \\
\hline
\end{tabular}

Para os modelos calculados com intervalos de classe, as variáveis das equações apresentaram graus de correlação $(R)$ bastante satisfatórios, tendo sido verificado um sensível acréscimo nos valores dos mesmos. Os graus de determinação $\left(R^{2}\right)$ calculados demonstraram que existe uma elevada proporção entre as variáveis dependentes e a variáveis independentes.

Os graus de associação calculados para os modelos 11 a 20, com intervalo de classe, são apresentados na Tabela 10, a seguir.

TABELA 10 - GRAUS DE ASSOCIAÇÃO, MODELOS DE 11 A 20

\begin{tabular}{c|c|c|c|c}
\hline Modelo & $\mathbf{R 1}$ & Grau de R1 & $\mathbf{R 1}^{\mathbf{2}}$ & Grau de R1 $^{\mathbf{2}}$ \\
\hline 11 & 0,98 & Muito forte & 0,97 & Muito forte \\
\hline 12 & 0,98 & Muito forte & 0,97 & Muito forte \\
\hline 13 & 0,95 & Muito forte & 0,91 & Muito forte \\
\hline 14 & 0,93 & Muito forte & 0,87 & Forte \\
\hline 15 & 0,89 & Forte & 0,79 & Forte \\
\hline 16 & 0,88 & Forte & 0,78 & Forte \\
\hline Modelo & $\mathbf{R 2}$ & Grau de R2 & $\mathbf{R 2}^{\mathbf{2}}$ & Grau de R2 \\
\hline 17 & 0,95 & Muito forte & 0,90 & Forte \\
\hline 18 & 0,93 & Muito forte & 0,87 & Forte \\
\hline 19 & 0,86 & Forte & 0,73 & Forte \\
\hline 20 & 0,87 & Forte & 0,75 & Forte \\
\hline
\end{tabular}

Legenda: Coeficiente de correlação linear simples (R1); coeficiente de correlação linear múltipla (R2); coeficiente de determinação linear simples $\left(\mathrm{R} 1^{2}\right)$; coeficiente de determinação linear múltipla $\left(\mathrm{R} 2^{2}\right)$. 
Os modelos sem intervalos de classe foram submetidos ao teste estatístico de Análise da Variância pela distribuição F-Normal de Fisher-Snedecor para verificação da significância de seus regressores, por terem apresentado graus de associação "Fraca" e "Intermediária". Esta análise demonstrou que as variáveis independentes adotadas contribuem para a formação da variável dependente e seus regressores são significantes.

$\mathrm{Na}$ Tabela 11, a seguir, são apresentados os resultados de Análise da Variância, ao nível de significância de $5 \%$, para os modelos 1 a 10 , onde a condição de validação é Fcalc>Ftab.

TABELA 11 - ANÁLISE DA VARIÂNCIA, MODELOS 1 A 10

\begin{tabular}{c|c|c|c}
\hline Modelo & Fcalc & Ftab (5\%) & Situação \\
\hline 1 & 324,179 & 2,0283 & Fcalc $>$ Ftab \\
\hline 2 & 1052,739 & 1,4149 & Fcalc $>$ Ftab \\
\hline 3 & 56,203 & 2,0283 & Fcalc $>$ Ftab \\
\hline 4 & 196,531 & 1,4149 & Fcalc $>$ Ftab \\
\hline 5 & 26,401 & 2,0283 & Fcalc $>$ Ftab \\
\hline 6 & 103,403 & 1,4149 & Fcalc $>$ Ftab \\
\hline 7 & 13,234 & 2,0540 & Fcalc $>$ Ftab \\
\hline 8 & 14,349 & 2,0540 & Fcalc $>$ Ftab \\
\hline 9 & 16,711 & 1,4166 & Fcalc $>$ Ftab \\
\hline 10 & 16,831 & 1,4166 & Fcalc $>$ Ftab \\
\hline
\end{tabular}

A Tabela 12, a seguir, apresenta alguns modelos de uso corrente na literatura para o cálculo dos níveis sonoros em rodovias. 
Avaliação do ruído de tráfego noturno - estudo de caso na cidade de Curitiba, Brasil

TABELA 12 - MODELOS MATEMÁTICOS CONSOLIDADOS NA LITERATURA

\begin{tabular}{|c|c|c|}
\hline Modelo & \multicolumn{2}{|l|}{ Equação } \\
\hline $\begin{array}{l}\text { LANGDON e } \\
\text { SCHOLES } \\
\text { (1968) }\end{array}$ & $L_{e q}=10 \log \left[\frac{1}{T} \int_{0}^{T} 10^{\left(L_{T} / 10\right)} d T\right.$ & \\
\hline $\begin{array}{c}\text { GARCÍA e } \\
\text { FAUS (1991) }\end{array}$ & \multicolumn{2}{|l|}{$L_{e q}=48,6+8,1 \log Q$} \\
\hline $\begin{array}{l}\text { SANCHO e } \\
\text { SENCHERMES } \\
(1983)\end{array}$ & \multicolumn{2}{|c|}{$L_{10}=16 \log v+9 \log Q+0,120 H+18$} \\
\hline CoRTN (1988) & $L_{10}=10 \log q+33 \log [v+40+(500 / v)]+10 \log [1+$ & $+0,3 G-27,6$ \\
\hline $\begin{array}{l}\text { SANCHO e } \\
\text { SENCHERMES } \\
(1983)\end{array}$ & \multicolumn{2}{|c|}{$L_{50}=13 \log v+15 \log Q+0,010 H-3$} \\
\hline STEELE (2001) & \multicolumn{2}{|l|}{$L_{50}=68+\log V-20 \log D$} \\
\hline $\begin{array}{l}\text { SANCHO e } \\
\text { SENCHERMES } \\
(1983)\end{array}$ & \multicolumn{2}{|c|}{$L_{90}=10 \log v+21 \log Q+0,075 H-24$} \\
\hline $\begin{array}{l}\text { ALVES FILHO } \\
(1997)\end{array}$ & \multicolumn{2}{|l|}{$L_{90}=52,08+2,80 \log Q L$} \\
\hline
\end{tabular}

Nota: Todos os modelos elaborados a partir de valores medidos e com regressão logarítmica, bem como, sem intervalo de classe.

Fonte: Paz, 2004.

Da análise das Tabelas 7, 9 e 12, verifica-se que os modelos propostos sobressaem e diferem da maioria dos modelos existentes na literatura em duas características: 1) A linearidade dos modelos e 2) A aplicação de intervalos de intervalos de classe.

\section{CONCLUSÕES}

Para o período noturno a composição do tráfego pode ser considerada equivalente, ou seja, a demanda de veículos leves se assemelha à demanda de veículos pesados.

Para amostras com $n \leq 30$, sem intervalos de classe, em vias semelhantes ao objeto da pesquisa, são válidos estatisticamente os modelos $1,3,5,7$ e 8 . Considerando os intervalos de classe, são válidos estatisticamente os modelos 11, $13,15,17$ e 18. 
Para amostras com $n>30$, sem intervalos de classe, em vias semelhantes ao objeto da pesquisa, são válidos estatisticamente os modelos 2, 4, 6, 9 e 10. Considerando os intervalos de classe, são válidos estatisticamente os modelos 12 , $14,16,19$ e 20.

Verificou-se que para amostras grandes $(n>30)$ e predição do valor do nível sonoro equivalente $\left(L_{e q}\right)$, a avaliação com intervalos de classe é a mais indicada.

Os modelos desenvolvidos neste trabalho apresentam como principais características:

- Aplicacão para o período noturno - Na literatura corrente, a grande maioria dos modelos estatísticos são desenvolvidos para o período diurno.

- A linearidade dos modelos - Na literatura corrente, a maioria dos modelos de predição acústica foram desenvolvidos utilizando regressão logarítmica, com exceção dos modelos desenvolvidos por García e Faus (1991) que utilizam regressão linear. Assim, os modelos desenvolvidos no presente trabalho apresentam menor complexidade de cálculo do que modelos desenvolvidos a partir de regressão logarítmica.

- A aplicacão de intervalos de classe - A maioria dos modelos de predição acústica para o ruído de tráfego são formulados em uma equação única, incluindo toda a faixa dinâmica de flutuação dos níveis de pressão sonora (níveis mais baixos de ruído até os níveis mais elevados). Contudo, em função da grande variabilidade nos níveis de ruído de tráfego, devido a complexidade da amostra - veículos leves (carros, motos, caminhonetes, etc), veículos pesados (caminhões, ônibus, etc.), estes modelos apresentam deficiência na predição sonora. O mesmo não ocorreu com modelos com intervalos de classe desenvolvidos para o período diurno, como comprovado por Prascevic et al. (1997) e Paz e Zannin (2010). Os modelos com intervalos de classe, desenvolvidos no presente trabalho, conseguiram caracterizar adequadamente o ruído de tráfego urbano para o período noturno. 


\section{AGRADECIMENTOS}

Os autores gostariam de agradecer ao DAAD - Deutscher Akademischer Austauschdienst (Serviço alemão de intercâmbio acadêmico), e ao e CNPq Conselho Nacional de Desenvolvimento Científico e Tecnológico - pelo apoio financeiro para o desenvolvimento desta pesquisa. Os autores gostariam de agradecer também, o trabalho dos revisores, com suas sugestões e críticas que ajudaram a melhorar as análises apresentadas no presente artigo.

\section{REFERÊNCIAS}

ALVES FILHO, J.M.; LENZI, A.; ZANNIN, P.H.T. Effects of traffic composition on road noise: a case study. Transportation Research Part D: Transport e Environment, v. 9(1), p. 75-80, 2004.

ASTOLFI, A.; CORRADO, V.; GRIGINIS, A. Comparison between measured e calculated parameters for the acoustical characterization of small classrooms. Applied Acoustics, v. 69, p. 966-976, 2008.

BURNS, W. Noise and Man. 1 ed. London: William Clowes e Sons, 236 p. 1973.

BURGESS, M.A. Noise Prediction for Urban Traffic Conditions - Related to Measurements in the Sydney Metropolitan Area. Applied Acoustic, v. 10, p. 1-7, 1977.

BELOJEVIC G.; JAKOVLJEVIC B. Subjective reactions to traffic noise with regard to some personal traits. Environment International, v. 23(2), p. 221-226, 1997.

BELOJEVIC, G.; JAKOVLJEVIC, B.; STOJANOV, V.; PAUNOVIC, K.; ILIC J. Urban road-traffic noise e blood pressure e heart rate in preschool children. Environment International, v. 34, p. 226-231, 2008.

CVETKOVIC, D.; PRASCEVIC, M.; STOJANOVIC, V.; MIHAJLOV, D. Comparative Analysis of Traffic Noise Prediction Models. $5^{\text {th }}$ Congress of Slovenian Acoustical Society, Solovenian, p. 349-357, 1998.

CALIXTO, A.; DINIZ F.B.; ZANNIN, P.H.T. The statistical modeling of road traffic noise in an urban setting. Cities, v. 20(1), p 23-29, 2003.

CALIXTO A.; PULCIDES, C.; ZANNIN, P.H.T. Evaluation of transportation noise in urbanized areas - A case study. Archives of Acoustics, v. 33(2), p. 151-164, 2008. 
DANTAS, G.H.G. Manual de Pavimentação. 2 ed. Rio de Janeiro: DNER, 320 p., 1996.

DER BUNDESMINISTER FÜR VERKEHR - DBV. RLS/90: RICHTLINIEN FÜR DEN LÄRMSCHUTZ AN STRAßEN (Diretrizes para Proteção ao Ruído em Rodovias). Bonn, (em alemão) 1990.

DÍAZ, C.; PEDRERO, A. Sound exposure during daily activities. Applied Acoustics, v. 67, p. $271-283,2006$.

FERNÁNDEZ, M.; QUINTANA, S.; CHAVARRÍA, N.; BALLESTEROS, J.A. Noise exposure of workers of the construction sector. Applied Acoustics, v. 70(5), p. 753760, 2009.

GARCÍA A.; FAUS L.J. Statistical Analysis of Noise Levels in Urban Areas. Applied Acoustics, v. 3(91), p. 227-247, 1991.

GERGES, S.N.Y. Noise in large cities in Brazil. Journal of the Acoustical Society of America, v. 115(5), p. 2592-2593, 2004.

GOLEBIEWSKI, R.; MAKAREWICK, R.; NOWAK, M.; PREIS, A. Traffic Noise Reduction due the porous Road Surface. Applied Acoustics, v. 64, p. 481-494, 2003.

GOLMOHAMMADI, R.; ABBASPOUR, M.; NASSIRI, P.; MAHJUB, H. Road traffic noise model. J Res Health Sci, v. 7(1), p. 13-17, 2007.

GUEDES, I.C.M.; BERTOLI, S.R.; ZANNIN P.H.T. Influence of urban shapes on environmental noise: A case study in Aracaju Brazil. Science of the Total Environment, v. 412, p. 66-76, 2011.

HAKA, M.; HAAPAKANGAS, A.; KERÄNEN, J.; HAKALA, J.; KESKINEN, E.; HONGISTO, V. Performance effects e subjective disturbance of speech in acoustically different office types - a laboratory experiment. Indoor Air, v. 19, p. 454467, 2009.

JAKOVLJEVIC, B.; PAUNOVIC, K.; BELOJEVIC, G. Road-traffic noise e factors influencing noise annoyance in an urban population. Environment International, v. 32, p. 552-556, 2009. 
KLATTE, M.; HELLBRÜCK, J. Effects of classroom acoustics on performance e wellbeing in elementary school children: A field study In: Inter Noise, June, Lisbon, Portugal, 2010.

MAKAREWICZ, R. A Simple Model of Outdoor Noise Propagation. Applied Acoustic, v. 54, n. 2, p. 131-140, 1998.

MASCHKE, C. Preventive Medical Limits for Chronic Traffic Noise Exposure. Acustica v. 85, p. 448-461, 1999.

MAKAREWICZ, R.; FUJIMOTO, M.; KOKOWSKI, P. A Model of Interrupted Road Traffic Noise. Applied Acoustic, v. 57, p. 129-137, 1999.

MAUTE, D. Technische Akustik und Lärmschutz. Fachbuchverlag Leipzig im Carls Hanser Verlag, Deutschland (em Alemão), 2006.

MUZET, A. Environmental noise, sleep e health. Sleep Medicine Reviews, v. 11, p. 135-142, 2007.

NATIONAL GEOGRAPHIC MAGAZINE - National Geographic Society, ano 11, number 130, Januar 2011.

PRASCEVIC, M.R.; CVETKOVIC, D.S.; DELJANIN, A.S.; STOJANOVIC, V.O. Modeling of Urban Traffic Noise. $5^{\text {th }}$ International Congress on Sound e Vibration, Australia, p. 1-6, 1997.

PAZ, E.C. Estudo de um Modelo de Avaliação e Predição para o Ruído de Tráfego, Curitiba. 2004. Dissertação (Mestrado em Construção Civil), Universidade Federal do Paraná, $417 \mathrm{p}$.

PAZ, E.C.; FERREIRA, A.M.C.; ZANNIN, P.H.T. Estudo comparativo da percepção do ruído urbano. Rev Saúde Pública, v. 39(3), p. 467-72, 2005.

PORTELA, B.S.; ZANNIN, P.H. Analysis of factors that influence noise levels inside urban buses. J Scientific and Industrial Research, v.69, p. 684-7, 2010.

PAZ, E.C.; ZANNIN, P.H.T. Urban daytime traffic noise prediction models. Environmental Monitoring e Assessment, v. 163, p. 515-529, 2010.

PASSERO, C.R.M.; ZANNIN, P.H.T. Statistical comparison of reverberation times measured by the integrated impulse response $e$ interrupted noise methods, 
computationally simulated with ODEON software, e calculated by Sabine, Eyring e Arau-Puchades' formulas. Applied Acoustics, v. 71, p. 1204-1210, 2009.

PAZ, E.C.; ZANNIN, P.H.T. Avaliação da poluição sonora no Campus III - Centro Politécnico e Campus Jardim Botânico da Universidade Federal do Paraná Curitiba, PR. RA'EGA, v. 26, p. 5-34, 2012.

ROMEU, J.; GNESCÀ, M.; PÀMIES, T.; JIMÉNEZ, S. Street categorization for the estimation of day levels using short-term measurements. Applied Acoustics, v. $72(8)$, p. 569-577, 2011.

SEBERG, U. Road Traffic Noise - The Influence of the Road Surface e its Characterization. Applied Acoustics, v. 21, p. 97-118, 1987.

SATTLER, M.A. Urban Noise Survey for the city Porto Alegre, Brazil. $137^{\text {th }}$ Meeting of the Acoustical Society of America - Fórum Acusticum, Berlim, p. 1-6, 1999.

SUKSAARD, T.P.; SUKASEM, P.; TABUCANON, S.M.; AOI, I.; SHIRAI, K.; TANAKA, T. Road Traffic Noise Prediction model in Thailand. Applied Acoustic, v. 58, p. 123-130, 1999.

STEELE, C.A. Critical Review of Some Traffic Noise Prediction Models. Applied Acoustic, v.62, p. 271-287, 2001

SZEREMETA, B.; ZANNIN, P.H.T. Analysis e evaluation of soundscapes in public parks through interviews e measurement of noise. Science of the Total Environment , v. 407, p. 6143-6149, 2009.

TANG, S.K.; TONG, K.K. Estimating traffic noise for inclined roads with freely flowing traffic. Applied Acoustics, v. 65, p. 171-181, 2004.

TO, W.M.; RODNEY, C.; IP, W. A multiple regression model for urban traffic noise in Hong Kong. Journal of the Acoustical Society of America, v. 112, n. 2, p. 551556, 2002.

VERBAND DER AUTOMOBILINDUSTRIE E. V. - VDA. Urban Traffic e Noise. 1 ed. Frankfurt: VDA, 135 p., 1978.

ZANNIN, P.H.T.; CALIXTO, A.; DINIZ, F.B.; FERREIRA, J.A.; SCHUHLI R. Incômodo causado pelo ruídourbano à população de Curitiba. Revista de Saúde Pública, v. 36(4), p. 521-524, 2002. 
ZANNIN, P.H.T.; DINIZ, F.B.; FERREIRA, J.A.C. Interior noise profiles of buses in Curitiba. Tansportation Research Par D - Transport and Environment, v. 8(3), p. 243-247, 2003.

ZANNIN, P.H.T.; SZEREMETTA, B. Avaliação da poluição sonora no parque Jardim Botânico de Curitiba, Paraná, Brasil. Cadernos de Saúde Pública, v. 19(2), p. 683686, 2003.

ZANNIN, P.H.T.; FERREIRA, A.M.C.; SZEREMETA, B. Evaluation of noise pollution in urban parks. Environmental Monitoring and Assessment, v. 118, p. 423-33, 2006.

ZANNIN, P.H.T.; MARCON, C.R. Objective e subjective evaluation of the acoustic comfort in classrooms. Applied Ergonomics, v. 38, p. 675-680, 2007.

ZANNIN, P.H.T. Occupational noise in urban buses. International Journal of Industrial Ergonomics, v. 38 (2), p. 232-237, 2008.

ZANNIN, P.H.T.; ZWIRTES, D.P.Z. Evaluation of the acoustic performance of classrooms in public schools. Applied Acoustics, v. 70, p. 626-635, 2009.

ZANNIN, P.H.T.; FERREIRA, A.M.C. Field measurements of acoustic quality in university classrooms. Journal of Scientific e Industrial Research, v. 68, p. 10531057, 2009.

ZANNIN, P.H.T.; SANT'ANA, D.Q. Noise mapping at different stages of a freeway redevelopment project - A case study in Brazil. Applied Acoustics, v. 72(8), p. 479486, 2011.

ZANNIN, P.H.T.; ENGEL, M.S.; FIEDLER, P.E.K.; BUNN, F. Characterization of environmental noise based on noise measurements, noise mapping and interviews: a case study at a university campus in Brazil. Cities, v. 31, p. 317-327, 2013. 